\title{
The role of the density gradient on intermittent cross-field transport events in a simple magnetized toroidal plasma
}

\author{
C. Theiler, ${ }^{1}$ A. Diallo, ${ }^{1}$ A. Fasolii, ${ }^{1}$ I. Furno, ${ }^{1}$ B. Labit, ${ }^{1}$ M. Podestà, ${ }^{1,2}$ F. M. Polii, ${ }^{1,3}$ \\ and P. Ricci ${ }^{1}$ \\ ${ }^{1}$ Centre de Recherches en Physique des Plasmas-Ecole Polytechnique Fédérale de Lausanne, \\ Association EURATOM-Confédération Suisse, CH-1015 Lausanne, Switzerland \\ ${ }^{2}$ Department of Physics and Astronomy, University of California, Irvine, California 92697, USA \\ ${ }^{3}$ Center for Fusion, Space and Astrophysics, Department of Physics, University of Warwick, \\ Coventry CV4 7AL, United Kingdom
}

(Received 6 December 2007; accepted 29 February 2008; published online 11 April 2008)

\begin{abstract}
Intermittent cross-field particle transport events (ITEs) are studied in the basic toroidal device TORPEX [TORoidal Plasma EXperiment, A. Fasoli et al., Phys. Plasmas 13, 055902 (2006)], with focus on the role of the density gradient. ITEs are due to the intermittent radial elongation of an interchange mode. The elongating positive wave crests can break apart and form blobs. This is not necessary, however, for plasma particles to be convected a considerable distance across the magnetic field lines. Conditionally sampled data reveal two different scenarios leading to ITEs. In the first case, the interchange mode grows radially from a slab-like density profile and leads to the ITE. A novel analysis technique reveals a monotonic dependence between the vertically averaged inverse radial density scale length and the probability for a subsequent ITE. In the second case, the mode is already observed before the start of the ITE. It does not elongate radially in a first stage, but at a later time. It is shown that this elongation is preceded by a steepening of the density profile as well. (C) 2008 American Institute of Physics. [DOI: 10.1063/1.2901188]
\end{abstract}

\section{INTRODUCTION}

Intermittency in cross-field particle transport is reported from the plasma edge of virtually all laboratory devices and is an active research area of plasma confinement. ${ }^{1-11} \mathrm{~A}$ better understanding of intermittent cross-field transport could help in finding ways to control it and to increase the performance of confinement devices. Basic plasma devices, which feature a better diagnostic accessibility than fusion devices, allow detailed experimental studies of intermittent cross-field transport and provide a test bed for numerical simulations.

The experiments reported here are performed in the basic toroidal device TORPEX. ${ }^{12}$ The plasma is produced and sustained by microwaves in the electron cyclotron (EC) range of frequencies. Typical plasma parameters are electron density $n \leqslant 10^{17} \mathrm{~m}^{-3}$, electron temperature $T_{e} \approx 5 \mathrm{eV}$, ion temperature $T_{i} \ll T_{e}$, and $\beta \ll 1$, where $\beta$ is the ratio of thermal pressure to magnetic pressure. The magnetic field configuration consists of a toroidal field on which a vertical field component is superimposed. This leads to helical magnetic field lines that intercept the vacuum vessel at the bottom and the top.

Recently, a new configuration has been achieved in TORPEX, which features a high level of intermittent crossfield transport of particles. ${ }^{13}$ On the low-field side (LFS), measurements with the two-dimensional (2D) Langmuir probe (LP) array HEXTIP ${ }^{14}$ covering most of the plasma cross section showed the formation and propagation of plasma blobs. The latter are propagating structures of increased plasma density, which are extended along the magnetic field lines, but localized in the perpendicular plane. They form from a radially elongated interchange wave. Their statistical properties studied with a pattern-recognition method show strong similarities to blob observations in tokamaks. ${ }^{13}$

The generation mechanism of blobs has been identified in a similar configuration by a conditional sampling (CS) technique, using time resolved, two-dimensional profiles of density, electron temperature and plasma potential. ${ }^{15} \mathrm{~A}$ radially sheared vertical $\mathbf{E} \times \mathbf{B}$ flow breaks apart radially elongated wave structures, generating blobs. A local steepening of the pressure profile is observed to precede the radial elongation of the wave.

In this paper, the reason for the radial elongation of the mode is further studied by operating HEXTIP in the ion saturation current regime. Simultaneous measurements over the plasma cross section allow not only the reconstruction of the conditionally averaged dynamics of intermittent cross-field particle transport events (ITEs), but also the analysis of individual events, and the use of nonlocal trigger conditions for conditional sampling.

Individual snapshots from HEXTIP show that isolated peaks detected at a single probe on the LFS can be caused by detached structures (blobs) as well as by radially elongated structures. In a first step, conditional sampling ${ }^{16}$ is applied to two vertically separated reference probes on the LFS. This gives different average dynamics of ITEs at the two positions, indicating that the density varies along the magnetic field lines.

ITEs detected at the lower of the two reference probes originate predominantly from a steep density profile. A novel analysis technique reveals a monotonic dependence between the vertically averaged inverse radial density scale length and the probability for a subsequent ITE. The conditionally av- 
eraged dynamics leading to ITEs at the upper reference probe, on the contrary, reveal a different scenario. The mode is already observed before the start of the ITE. It does not grow radially in a first stage, but at a later time. We show that this sudden radial elongation of the positive wave crest is preceded by a steepening of the density profile as well. This is done by comparing the dynamics leading to ITEs with the average evolution of the mode.

This paper is organized as follows. In Sec. II, we describe the experimental setup and the basic properties of the investigated plasma. The different conditionally averaged dynamics leading to ITEs at the two vertically separated reference probes are presented in Sec. III. The role of the density gradient for ITEs at the lower probe is studied in Secs. IV and $\mathrm{V}$, and that for the upper probe is studied in Sec. VI. The results are summarized in Sec. VII.

\section{EXPERIMENTAL SETUP AND DIAGNOSTICS}

The experiments are performed in the simple magnetized toroidal device TORPEX (major radius $R=1 \mathrm{~m}$, minor radius $a=0.2 \mathrm{~m}$ ) using hydrogen plasmas produced and sustained by means of microwaves in the EC range of frequencies. ${ }^{17}$ A microwave power $P_{R F}=400 \mathrm{~W}$ is used in a toroidal magnetic field $B_{\text {tor }}=74.4 \mathrm{mT}$ on which a vertical magnetic field $B_{z}=2.4 \mathrm{mT}$ is superimposed. The neutral gas pressure is set to $3.5 \times 10^{-5}$ mbar. Measurements are taken with the twodimensional LP array HEXTIP ${ }^{14}$ biased in the ion saturation current regime. This diagnostics covers most of the plasma cross section with a spatial resolution of $3.5 \mathrm{~cm}$ and data are acquired at a sampling frequency of $250 \mathrm{kHz}$. The density is deduced from the ion saturation current by assuming a constant electron temperature of $5 \mathrm{eV}$. When not otherwise stated, the analysis in this paper is based on 12 identical $1.5 \mathrm{~s}$ discharges.

The combination of a relatively strong vertical field component and a low injected microwave power results in a vertically elongated plasma profile located at the high-field side (HFS), Fig. 1(a). A high level of relative density fluctuations is observed in the bad curvature region, where the magnetic field gradient and the density gradient are collinear, Fig. 1(b).

Most of the ionization occurs at the EC resonance and at the upper hybrid (UH) resonance. ${ }^{17}$ While the EC layer lies at $r \approx-15 \mathrm{~cm}$, the location of the $\mathrm{UH}$ resonance depends on the instantaneous density. In order to estimate the average location of the plasma source, the technique introduced in Ref. 17 is applied. We perform 10 discharges of $1.5 \mathrm{~s}$ each, where microwave pulses of amplitude $1.1 \mathrm{~kW}$ and duration $\approx 80 \mu$ s are superimposed to the constant injected power of $400 \mathrm{~W}$. The average $2 \mathrm{D}$ density profile measured at times well separated from any microwave pulse is then subtracted from the average profile measured at a certain time after the start of a pulse. The result is shown in Fig. 1(c). It shows the effect $\Delta n$ of the microwave pulses on the average density profile $16 \mu$ s after the beginning of a pulse and is the average over 5730 events. We find $\Delta n \approx 0$ for $r>0$, which indicates that the LFS is essentially source-free.

The bad curvature side of the source region is dominated by a strong mode of frequency $f \approx 4.5 \mathrm{kHz}$ in the laboratory frame, in which it propagates upwards due to an $\mathbf{E} \times \mathbf{B}$ flow along the $z$ direction. ${ }^{15}$ The mode location is indicated in Fig. 1(e), where the profile of the spectral power of $\widetilde{n}$ in the frequency band $3.5-5.5 \mathrm{kHz}$ is shown. In Fig. 1(f), we show the power spectral density of $\tilde{n}$ measured at the three probe tips indicated in (e). Such waves have been identified as drift-interchange waves in TORPEX via a full reconstruction of the dispersion relation in all three directions. They have a strong interchange character for high values of $B_{z}{ }^{18,19}$

The profile of density skewness in Fig. 1(d) is used to illustrate the nature of fluctuations in the different plasma regions. Negatively skewed signals are found on the HFS of the mode in the region of largest mean density, indicating the presence of negative bursts. Conditional sampling performed by triggering on these negative bursts shows that they are mainly due to inward propagating negative cells of the mode. The positively skewed signals found in the LFS region are due to ITEs taking the form of elongated positive wave crests or blobs. When not otherwise stated, we show $\tilde{n}=n-\langle n\rangle_{t}$ in the following, i.e., the fluctuation of density around its time-averaged value. We note, however, that $\tilde{n}$ is not small compared to $n$; see Fig. 1(b).

\section{SPATIOTEMPORAL ITE DYNAMICS FROM CONDITIONAL SAMPLING}

In this section, we investigate the two-dimensional spatiotemporal dynamics of ITEs using conditional sampling. ${ }^{16}$ HEXTIP tips $P_{1}$ and $P_{2}$ indicated in Fig. $1(\mathrm{~d})$ are separately used as reference probes. The two tips lie well outside the source region on the LFS at $r=8.8 \mathrm{~cm}$, $z=-9.1 \mathrm{~cm}$, and $z=3.0 \mathrm{~cm}$, respectively. We note that $P_{1}$ and $P_{2}$ represent points characterized by different distances to the vacuum vessel. The shortest paths from $P_{1}$ and $P_{2}$ to the vessel wall along the magnetic field lines have a length of approximately $2.5 \mathrm{~m}$ and $4.3 \mathrm{~m}$, respectively. The connection length $L_{c}$ of the field lines is the same at both points $\left(L_{c} \approx 10.3 \mathrm{~m}\right.$ at $\left.r=8.8 \mathrm{~cm}\right)$.

We assume that every local maxima in the reference signal $\widetilde{n}_{P_{1,2}}$ that exceeds a certain threshold value corresponds to an ITE. The conditional average $\langle\tilde{n}\rangle_{\mathrm{ca}}(\mathbf{x}, \tau)$ of $\tilde{n}$ is evaluated as

$$
\langle\widetilde{n}\rangle_{\mathrm{ca}}(\mathbf{x}, \tau)=\frac{1}{N} \sum_{i=1}^{N} \widetilde{n}\left(\mathbf{x}, t_{i}+\tau\right),
$$

where $t_{i}$ is a time at which the trigger condition at the reference position $\left(P_{1}\right.$ or $\left.P_{2}\right)$ is satisfied and $N$ is the total number of triggered events. We use a threshold value of $3.5 \sigma$ as a trigger condition, where $\sigma$ is the standard deviation of the reference signal. This corresponds to a threshold of $\approx 1.1 \times 10^{16} \mathrm{~m}^{-3}$ for $\widetilde{n}_{P_{1}}$ and $\approx 1.2 \times 10^{16} \mathrm{~m}^{-3}$ for $\widetilde{n}_{P_{2}}$.

In addition to the conditional average, we also evaluate the reproducibility $C^{\text {rep }}$, which measures the deviation of individual events from the average. Following Ref. 20, it is defined by 

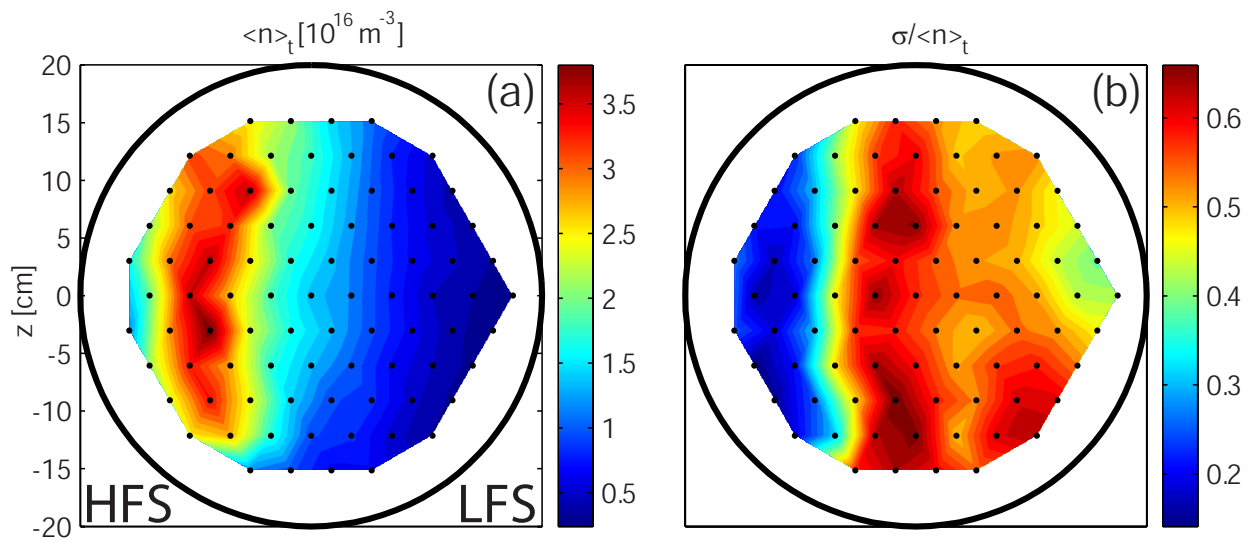

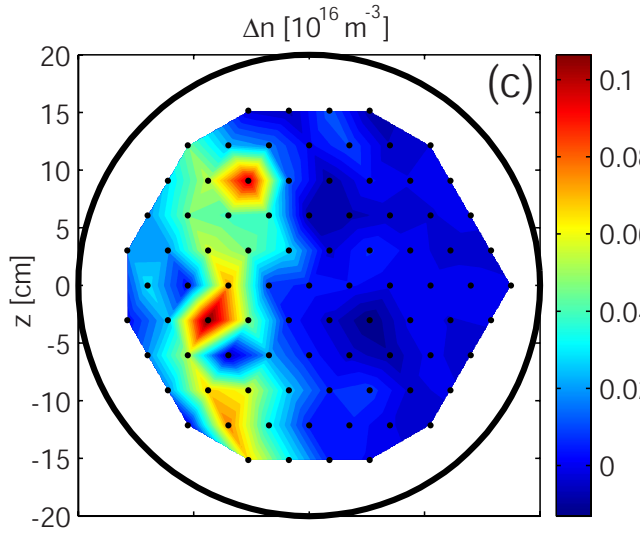

Spectral power between

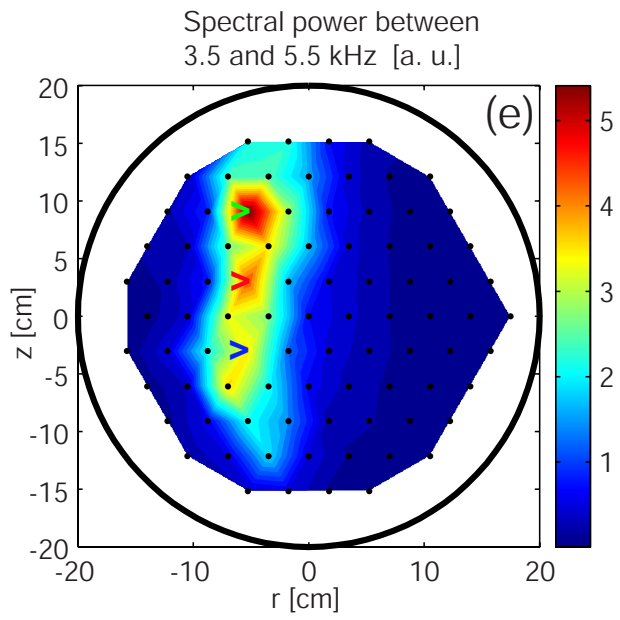

$$
C_{\mathrm{rep}}(\mathbf{x}, \tau) \equiv 1-\frac{\sigma_{\mathrm{ca}}^{2}(\mathbf{x}, \tau)}{\left\langle\widetilde{n}^{2}\right\rangle_{\mathrm{ca}}(\mathbf{x}, \tau)} \equiv \frac{\langle\widetilde{n}\rangle_{\mathrm{ca}}^{2}(\mathbf{x}, \tau)}{\langle\widetilde{n}\rangle_{\mathrm{ca}}^{2}(\mathbf{x}, \tau)+\sigma_{\mathrm{ca}}^{2}(\mathbf{x}, \tau)}
$$

with $\sigma_{\mathrm{ca}}^{2}(\mathbf{x}, \tau) \equiv\left\langle\left(\widetilde{n}-\langle\widetilde{n}\rangle_{\mathrm{ca}}\right)^{2}\right\rangle_{\mathrm{ca}}(\mathbf{x}, \tau)$. $C_{\text {rep }}$ lies between 0 and 1 , being high for a good reproducibility of the selected events.

Before turning to the conditional average of ITEs, we give in Figs. 2(a)-2(c) three snapshots of individual events at times when the trigger condition at $P_{2}$ is satisfied. The corresponding time traces of the density at $P_{2}$ are shown in Fig. 2(d). This shows that a peak in the density time history detected at a single probe tip on the LFS is not always associated with a detached structure (blob), as in frames (a) and (b), but it can also be caused by a radially elongated struc- ture, frame (c). The same behavior can be observed for ITEs detected at $P_{1}$. We conclude that the radially elongating positive structure of the mode, which is at the origin of ITEs, can break apart and form a blob, as observed in Ref. 15. However, this is not required for the plasma to be convected to tip $P_{1}$ or $P_{2}$. We can interpret this behavior in the frame of semianalytical blob models (see Ref. 21 and references therein), where the polarization mechanism of blobs depends on the vertical shape of the blob, but not on its radial form. It is therefore not surprising to observe both blobs and streamer-like structures. We remark that there is no strong difference from single point measurements between blobs and streamers. It is not true in general that streamers lead to wider peaks, as Fig. 2(d) suggests. 

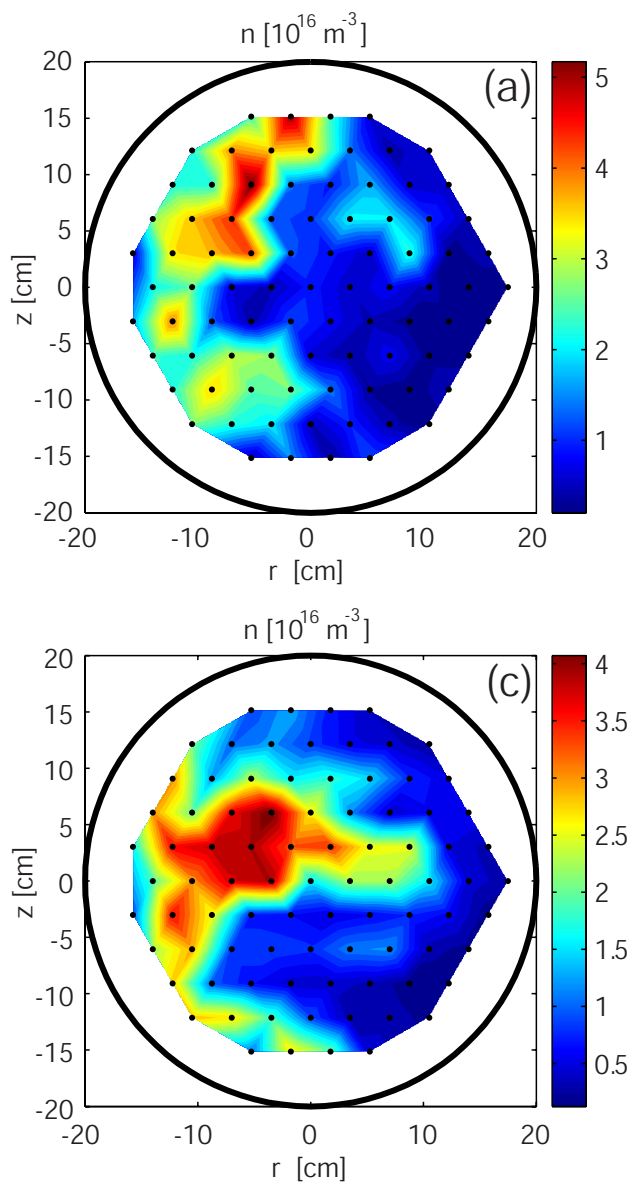
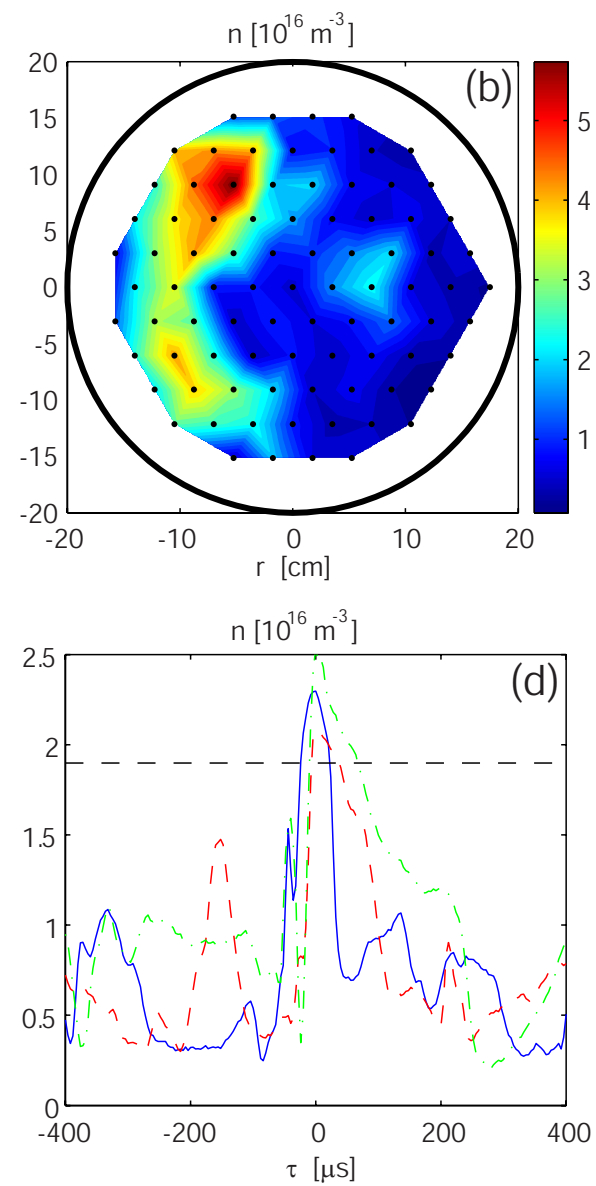

FIG. 2. (Color online) [(a)-(c)] Three examples of the instantaneous density profile at a time when the reference signal at $P_{2}$ satisfies the trigger condition. (d) Time traces of the total density $n$ at $P_{2}$ that correspond to the event in (a) (blue) to the one in (b) (dashed red) and to the one shown in (c) (dash-dotted green). The dashed horizontal line indicates the threshold value for $n_{P_{2}}$.
The conditionally averaged dynamics leading to ITEs at $P_{1}$ and $P_{2}$ as well as the reproducibility $C_{\text {rep }}$ are shown in Fig. 3 for five different values of time $\tau$, where $\tau=0$ corresponds to the time when the ITE is detected at $P_{1}$ or $P_{2}$.

The conditional average $\langle\tilde{n}\rangle_{\mathrm{ca}}$ for ITEs at $P_{1}$ (frames 1-5) shows a steep profile at $\tau=-160 \mu \mathrm{s}$, where the mode has a small amplitude. In the next two frames, a radial elongation of the mode is seen, which gives rise to the peak at $P_{1}$ in frame 5 imposed by the trigger condition. We should note that the reproducibility of the triggered events is poor in this case. This will be discussed in Sec. V.

In column 3 of Fig. 3, we show $\langle\widetilde{n}\rangle_{\text {ca }}$ for ITEs detected at $P_{2}$. It clearly differs from the previous case by the fact that the mode can already be distinguished at $\tau=-204 \mu \mathrm{s}$, frame 11, while there is no coherent signal for ITEs at $P_{1}$ at that time. At first, the mode does not grow radially. It rather seems to pull slightly back radially in frame 12 . It is only in frames 14 and 15 that the positive structure of the mode elongates radially, leading to the ITE. This is accompanied by an inward motion of the negative wave crests. Compared to ITEs detected at $P_{1}$, an improved reproducibility is observed for the positive and negative wave crests, as shown in column 4.

These results confirm that density bursts measured on the LFS are due to cross-field transport events originating on the HFS. The observed vertical wavelength of the mode, as well as the vertical distance of the two density peaks in frame 5 , are consistent with $\Delta$, the distance a field line moves upwards after one complete toroidal turn $\left(\Delta=2 \pi R B_{z} / B_{\text {tor }}\right.$ $\approx 20 \mathrm{~cm}$ ). This implies a uniform density along the magnetic field lines. However, the fact that the conditional average for ITEs at $P_{1}$ and $P_{2}$ differs by more than just a vertical translation indicates possible $3 \mathrm{D}$ effects in ITE dynamics. In the present paper, we consider only a poloidal cross section of the machine and we do not analyze the structures along the magnetic field lines.

\section{LINK BETWEEN THE INVERSE RADIAL DENSITY SCALE LENGTH AND ITES AT PROBE TIP $\boldsymbol{P}_{1}$}

The CS results in the preceding section suggest that ITEs detected at position $P_{1}$ are preceded by a steepened density profile. However, the low reproducibility found for the conditional average does not allow us to obtain more information from it. Motivated by numerical simulations of interchange turbulence in Ref. 22, we investigate the relation between the vertically averaged inverse radial density scale length $\left\langle L_{n}^{-1}\right\rangle_{z}(r, t) \equiv\langle 1 / n \times \partial n / \partial r\rangle_{z}(r, t)$ and the occurrence of ITEs. Note that $\left\langle L_{n}^{-1}\right\rangle_{z}(r, t)$ is defined in such a way that it is typically negative in the region of interest and strongly negative values correspond to steep profiles. In the following, we introduce and apply a technique that, contrary to the conditional average, is not based on an averaging process. This analysis technique can be used quite generally to investigate the relation between a certain condition on the plasma profile and the probability for an ITE. In a first step, the 

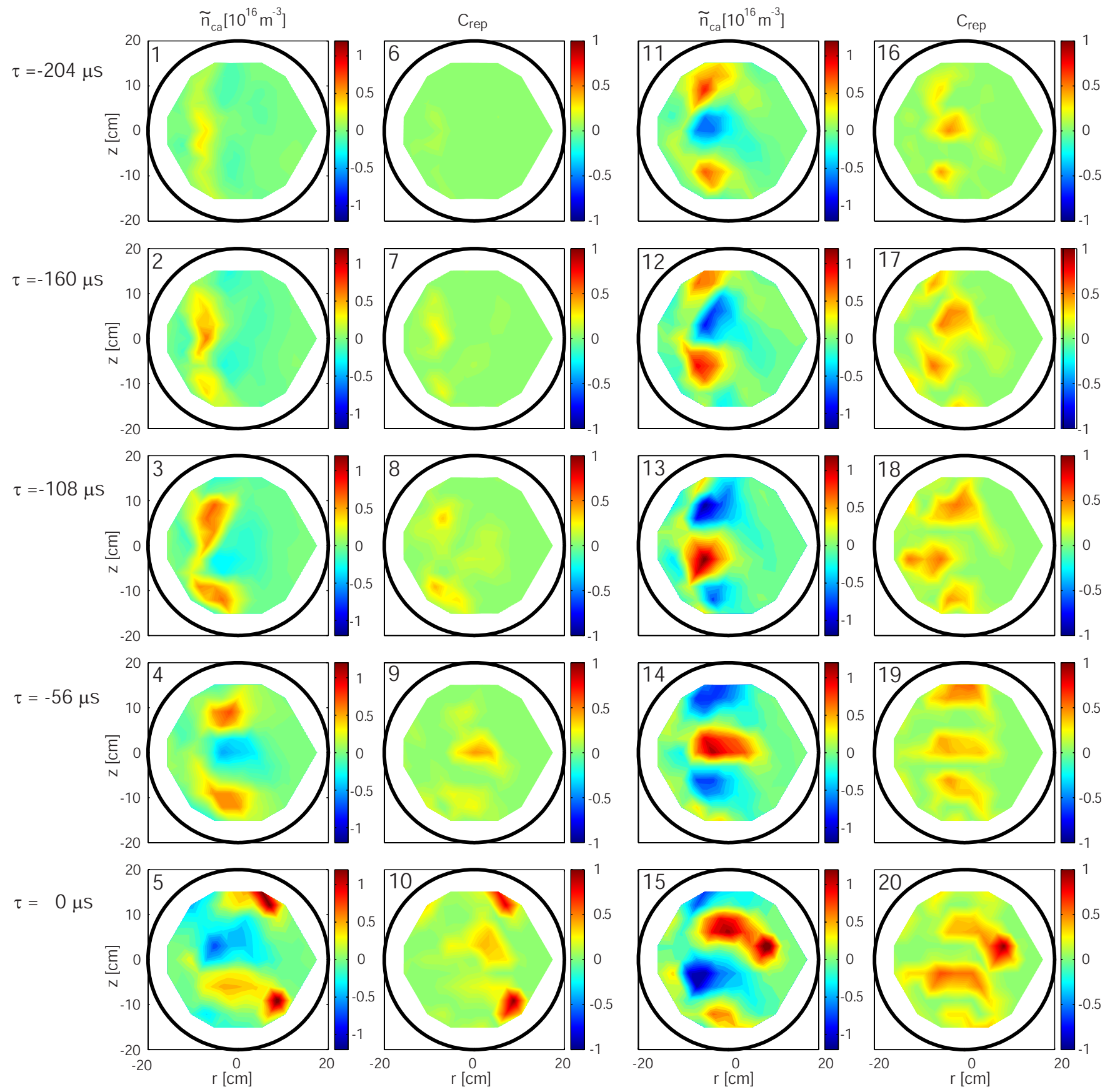

FIG. 3. (Color online) Column 1: Conditional average of $\tilde{n}$ obtained by triggering on ITEs at tip $P_{1}$ for five values of $\tau$. All local maxima exceeding 3.5 times the standard deviation of the signal were selected. Column 2: Reproducibility $C_{\text {rep }}$ of the conditional average in column 1 . Columns 3 and 4 : The same for ITEs at probe tip $P_{2}$.

signal $\left\langle L_{n}^{-1}\right\rangle_{z}(r, t)$ is searched for local minima that fall in a desired interval $\left[L_{l}^{-1}, L_{h}^{-1}\right]$. For every such event and different values of the time $\tau$ after these events, a signal $\widetilde{n}$ on the LFS is searched for the maximum value in a window of length $\Delta \tau$ around $\tau$, and this value is stored. All maxima exceeding a certain threshold value are interpreted as an ITE. In this way, for the local minima of $\left\langle L_{n}^{-1}\right\rangle_{z}(r, t)$ that fall in the interval $\left[L_{l}^{-1}, L_{h}^{-1}\right]$, we obtain the number of ITEs at a certain position on the LFS as a function of $\tau$ and the threshold value. By taking the maximum value of $\tilde{n}$ in the interval $[\tau-\Delta \tau / 2, \tau$ $+\Delta \tau / 2]$, we allow for a spread in the evolution time of ITEs.
In the following, $\Delta \tau=80 \mu \mathrm{s}$ is used. The results depend weakly on this parameter.

In order to establish the significance of the results obtained with this technique, the whole procedure is repeated for a "random" trigger, i.e., a trigger that arbitrarily selects events in the time series of $\left\langle L_{n}^{-1}\right\rangle_{z}(r, t)$. Finally, the results are assessed in a two-dimensional plot. The number of ITEs after a value of $\left\langle L_{n}^{-1}\right\rangle_{z}(r, t)$ in $\left[L_{l}^{-1}, L_{h}^{-1}\right]$ divided by the number of ITEs found by the random trigger (for the same number of initially triggered events) is plotted as a function of $\tau$ and the threshold value for ITEs. This two-dimensional plot, referred 

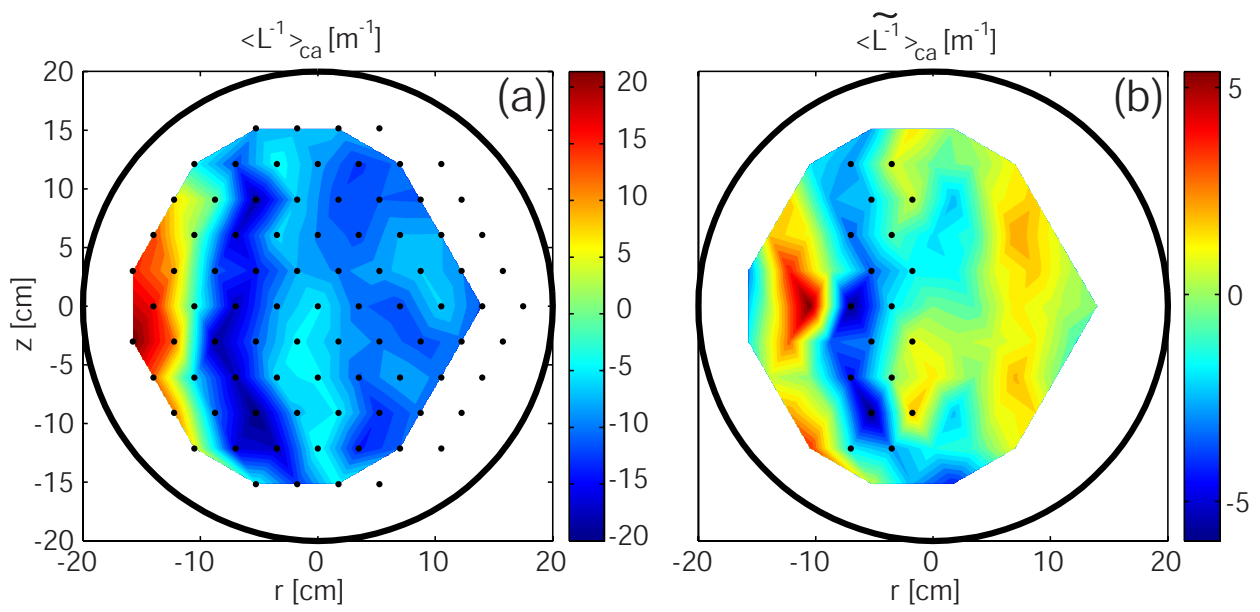

FIG. 4. (Color online) Conditionally averaged value of $L_{n}^{-1}$ (a) and its deviation $\left\langle\tilde{L}_{n}^{-1}\right\rangle_{\mathrm{ca}} \equiv\left\langle L_{n}^{-1}\right\rangle_{\mathrm{ca}}-\left\langle L_{n}^{-1}\right\rangle_{t}$ from the mean value (b) at $\tau=-160 \mu$ s before the ITEs are detected at $P_{1}$. The value of $\left\langle L_{n}^{-1}\right\rangle_{\mathrm{ca}}$ and $\left\langle\tilde{L}_{n}^{-1}\right\rangle_{\mathrm{ca}}$ evaluated between two neighboring tips is plotted at the position of the left tip. In (b) the pairs of tips used to evaluate $\left\langle L_{n}^{-1}\right\rangle_{z, 0}$ are indicated.

to as probability comparison chart (PCC) below, allows us to qualitatively evaluate how more likely it is to have an ITE after times when our condition on the plasma profile is fulfilled than at arbitrary times, and this as a function of time $\tau$ and of the threshold value defining ITEs.

The inverse radial density scale length is evaluated between two neighboring HEXTIP tips by

$$
L_{n}^{-1}=n^{-1} \frac{\partial n}{\partial r} \approx\left(\frac{n_{R}+n_{L}}{2}\right)^{-1} \times \frac{n_{R}-n_{L}}{\Delta r},
$$

where $n_{L}$ and $n_{R}$ are the instantaneous densities measured at the left and at the right tip and $\Delta r=3.5 \mathrm{~cm}$ is the tip separation. The choice of the radial position where we evaluate the vertical average of $L_{n}^{-1}(r, z, t)$ is motivated by the conditional average results shown in the preceding section. In Fig. 4, we show the conditionally averaged value of $L_{n}^{-1}$, as well as its deviation $\left\langle\tilde{L}_{n}^{-1}\right\rangle_{\mathrm{ca}} \equiv\left\langle L_{n}^{-1}\right\rangle_{\mathrm{ca}}-\left\langle L_{n}^{-1}\right\rangle_{t}$ from the mean value at $\tau=-160 \mu$ s, i.e., at the same time as frame 2 in Fig. 3. Negative values of $\left\langle\tilde{L}_{n}^{-1}\right\rangle_{\mathrm{ca}}$ are found in the region $r_{0} \approx-7 \mathrm{~cm}$. Guided by this, we estimate $\left\langle L_{n}^{-1}\right\rangle_{z}(r, t)$ around $r_{0}$ by averaging $L_{n}^{-1}$ over the values evaluated between the pairs of tips indicated in Fig. 4(b). We denote the result as $\left\langle L_{n}^{-1}\right\rangle_{z, 0}$ and we keep in mind that it is a function of time.

The analysis described above is then applied to the plasma scenario of interest. In Fig. 5(a), a subsample of the time trace of $\left\langle L_{n}^{-1}\right\rangle_{z, 0}$ is shown. We see that it fluctuates strongly and even assumes positive values. In Fig. 5(b), the histogram of local minima of $\left\langle L_{n}^{-1}\right\rangle_{z, 0}$ is shown. We only consider local minima that are a minimum in a time interval of $400 \mu$ s centered around each of them. This implies that the selected local minima are spaced by at least $200 \mu \mathrm{s}$, which is the typical evolution time of ITEs.

As a first step, we look at very strong minima in $\left\langle L_{n}^{-1}\right\rangle_{z, 0}$, i.e., at minima in the interval $\left[-33.5 \mathrm{~m}^{-1},-29.5 \mathrm{~m}^{-1}\right]$. More than 3000 such events are found. In Figs. 6(a) and 6(b), we show the conditional average $\langle\tilde{n}\rangle_{\mathrm{ca}}$ for these events at $\tau=0 \mu \mathrm{s}$ and at $\tau=52 \mu \mathrm{s}(\tau=0$ corresponds to the time of the local minima of $\left.\left\langle L_{n}^{-1}\right\rangle_{z, 0}\right)$. As anticipated, we find a steep profile at $\tau=0 \mu \mathrm{s}$. At $\tau=52 \mu \mathrm{s}$, the positive and negative wave crests are located at roughly the same vertical position as in frame 3 of Fig. 3.

The corresponding PCCs for probe tips $P_{1}$ and $P_{2}$ are shown in Figs. 6(c) and 6(d), respectively. The PCC for $P_{1}$ shows a clearly enhanced probability for ITEs for a time $\tau \approx 160 \mu \mathrm{s}$, which corresponds to the time expected from the conditional average as shown in the preceding section.

The PCC for $P_{2}$ shows an increased probability for ITEs at $\tau$ around $360 \mu \mathrm{s}$, however at a lower level than for $P_{1}$. We have evaluated the conditional average of $\tilde{n}$ for the events with a strong minimum of $\left\langle L_{n}^{-1}\right\rangle_{z, 0}$ that are followed by an ITE at $P_{2}$ (peak above $0.9 \times 10^{16} \mathrm{~m}^{-3}$ for $\tau \in[320 \mu \mathrm{s}, 400 \mu \mathrm{s}])$. The result showed that in these cases, the mode grows radially in a first step. It then stops extending further and gets convected upwards. It further elongates
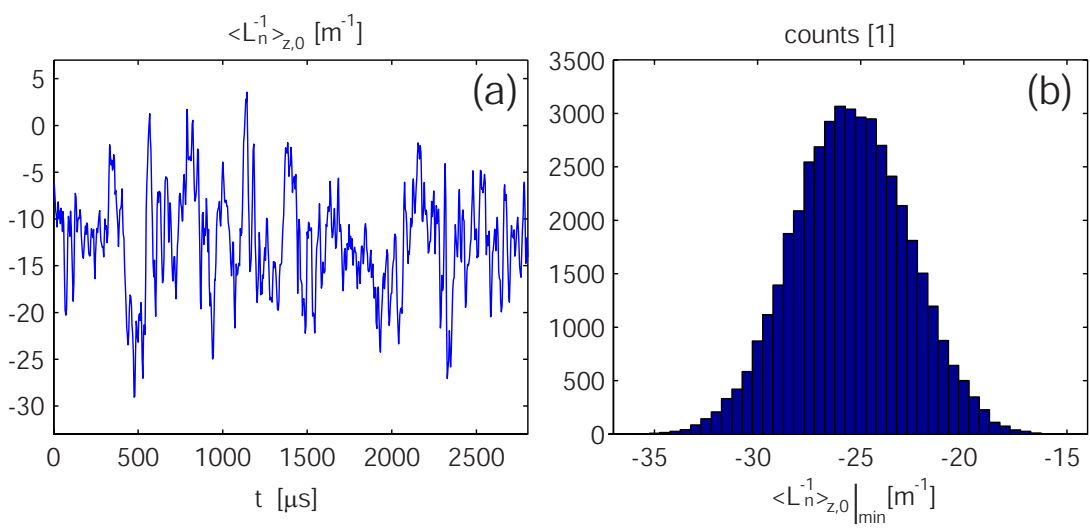

FIG. 5. (Color online) (a) Time trace of $\left\langle L_{n}^{-1}\right\rangle_{z, 0}$. (b) Histogram of local minima of $\left\langle L_{n}^{-1}\right\rangle_{z, 0}$. 

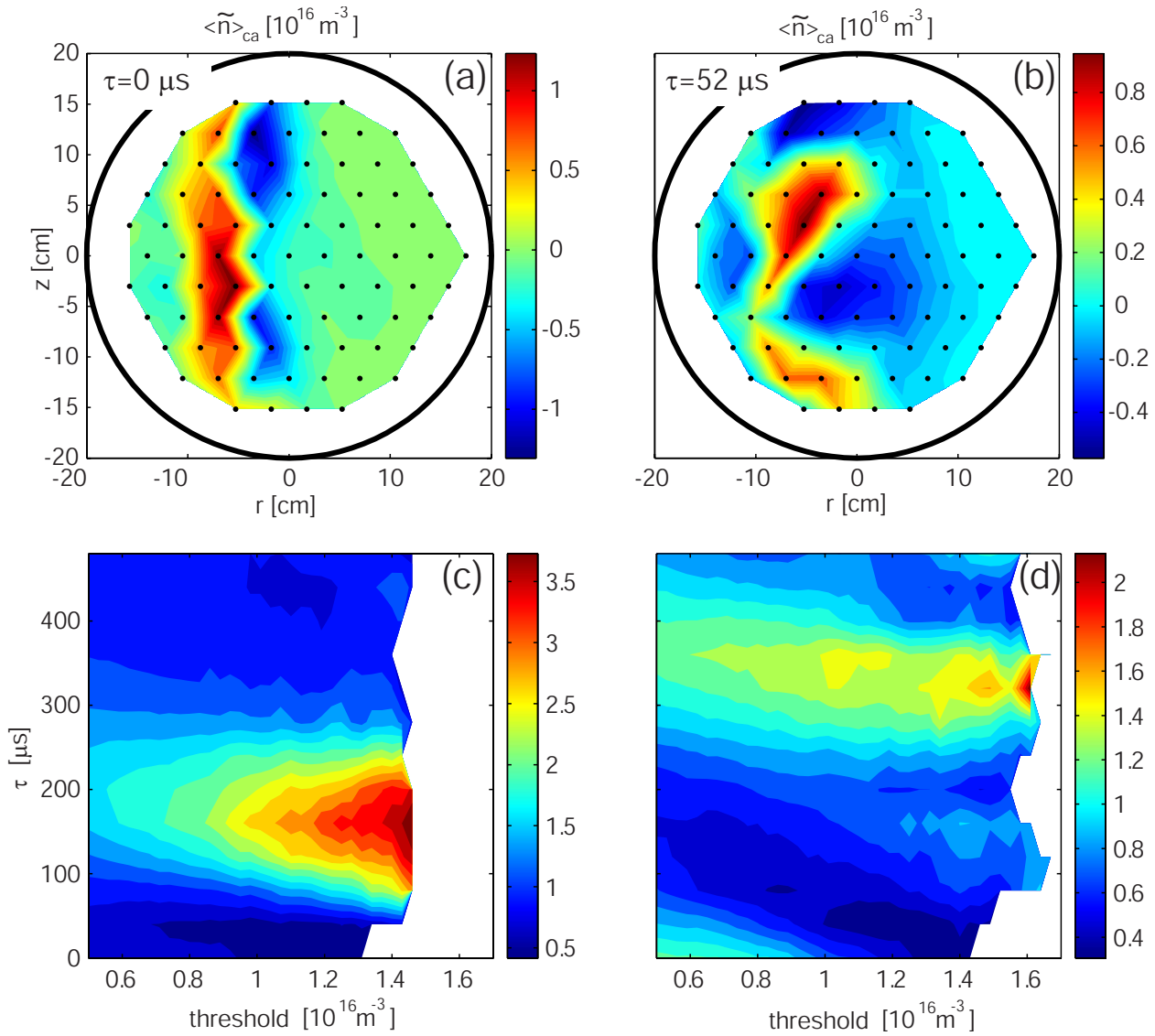

FIG. 6. (Color online) Results obtained by searching $\left\langle L_{n}^{-1}\right\rangle_{z, 0}$ for local minima in the lowest considered interval $\left[-33.5 \mathrm{~m}^{-1},-29.5 \mathrm{~m}^{-1}\right]$. [(a) and (b)] Conditional average of these events at $\tau=0$ and $\tau=52 \mu \mathrm{s}$, respectively. [(c) and (d)] Probability comparison chart for tip $P_{1}$ and $P_{2}$, respectively. The ratio of the number of ITEs after a strong negative value of $\left\langle L_{n}^{-1}\right\rangle_{z, 0}$ and the number after arbitrary chosen times is plotted as long as both values exceed 10 counts. at a much later time, in a similar way to that observed in the conditional average in Sec. III. From this, we conclude that the quantity $\left\langle L_{n}^{-1}\right\rangle_{z, 0}$ is not the one that governs the occurrence of ITEs at $P_{2}$. The reason a weak pattern can still be identified in the PCC is that the mode grows from a steep profile with a preferred phase.

We repeat now the analysis for subsequent intervals $\left[L_{l}^{-1}, L_{h}^{-1}\right]$. For each such interval, the PCC for $P_{1}$ looks similar to the one in Fig. 6(c), with a maximum number of ITEs for $\tau$ between 160 and $200 \mu$ s. In Fig. 7, this maximum divided by the number of triggered events in the corresponding interval is plotted for three different values of the threshold value defining ITEs. This gives an estimate of the probability for an ITE as a function of the preceding minimal value of $\left\langle L_{n}^{-1}\right\rangle_{z, 0}$. The result shows a monotonic dependence between $\left\langle L_{n}^{-1}\right\rangle_{z, 0}$ and the probability for an ITE. As a reference, we also show the ITE probability obtained for the randomly chosen times in the discharges (dashed lines).

\section{OBSERVATION OF THE TWO ITE SCENARIOS AT $\boldsymbol{P}_{1}$}

In the preceding section, we have seen that density profiles with a strongly negative value of $\left\langle L_{n}^{-1}\right\rangle_{z, 0}$ have an enhanced probability to give rise to ITEs at $P_{1}$. However, it is not clear whether a steep profile is a necessary condition for ITEs.

While the minimal values of $\left\langle L_{n}^{-1}\right\rangle_{z, 0}$ are well below its time average of $\approx-12 \mathrm{~m}^{-1}$ [see Fig. 5(b)], the results from CS in Fig. 4(b) show much smaller deviations. This could entirely be due to the reduction of amplitudes and therefore of gradients of a coherent structure introduced by the condi- tional averaging precess. This property of CS has been extensively studied in Ref. 23 with an analytical model, where amplitude reductions are due to errors in the determination of the exact occurrence time of a coherent structure due to noise (trigger jitter) as well as false trigger events, i.e., random fluctuations that give rise to a trigger event even though there is no coherent structure present.

In our case, besides false triggers, we expect to have a

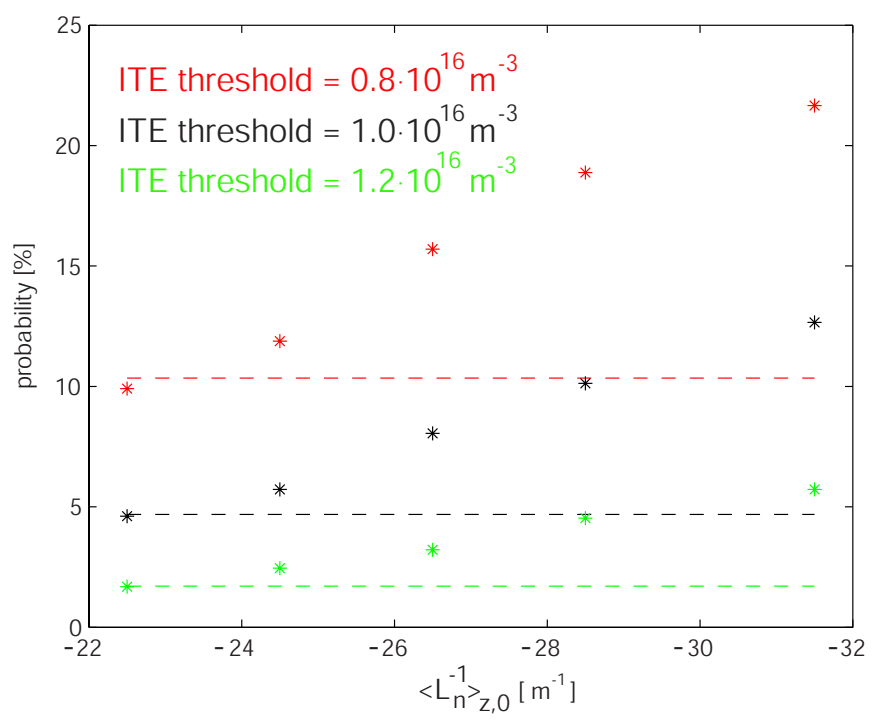

FIG. 7. (Color online) Probability for an ITE at $P_{1}$ as a function of the preceding minimal value of $\left\langle L_{n}^{-1}\right\rangle_{z, 0}$ for three different values above which a peak is interpreted as an ITE. The dashed lines show the corresponding probabilities for ITEs for arbitrarily chosen triggers in the time series. 

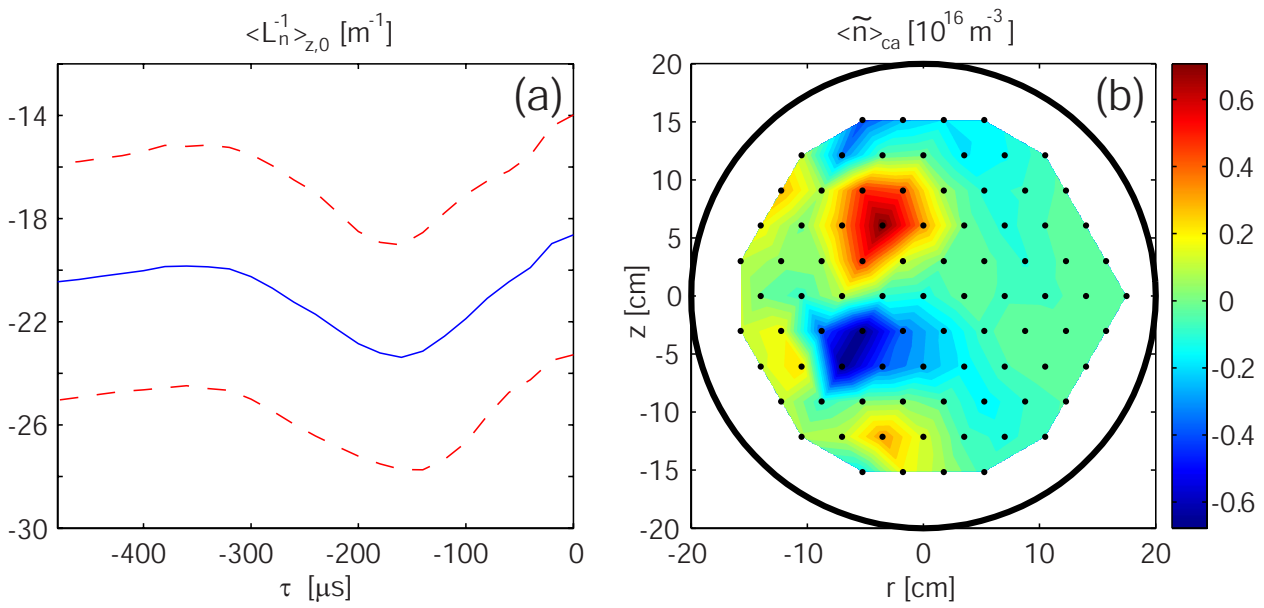

FIG. 8. (Color online) (a) Mean (blue line) and standard deviation (distance between blue and dashed red lines) of minimal values of $\left\langle L_{n}^{-1}\right\rangle_{z, 0}$ in a time window $\Delta \tau=80 \mu$ s centered around $\tau$ for all ITEs detected at $P_{1}$. (b) Conditional average $\langle\widetilde{n}\rangle_{\mathrm{ca}}$ at $\tau=-160 \mu \mathrm{s}$ of ITEs detected at $P_{1}$ that satisfy the additional constraint $\left\langle L_{n}^{-1}\right\rangle_{z, 0} \geqslant-21 \mathrm{~m}^{-1}$ for $\tau \in[-200 \mu \mathrm{s},-100 \mu \mathrm{s}]$.

dispersion in the time evolution of individual events, which has a similar effect to trigger jitter. To account for the latter in determining the minimal values of $\left\langle L_{n}^{-1}\right\rangle_{z, 0}$ involved in ITEs, we determine for each ITE the minimal value of $\left\langle L_{n}^{-1}\right\rangle_{z, 0}$ over a time window $\Delta \tau$ centered around a time $\tau$ before its detection at $P_{1}$ and we evaluate the mean value and the standard deviation of these minima. The results are shown in Fig. 8(a) for different values of $\tau$. As expected, we obtain the smallest values of $\left\langle L_{n}^{-1}\right\rangle_{z, 0}$ for $\tau \approx-160 \mu \mathrm{s}$. These values are closer to those encountered in the preceding section. Nevertheless, the result indicates that strongly negative values of $\left\langle L_{n}^{-1}\right\rangle_{z, 0}$ are not required for ITEs at $P_{1}$.

In order to determine if this is an effect of false triggers, we evaluate the conditional average of ITEs at $P_{1}$ that do not involve a strongly negative value of $\left\langle L_{n}^{-1}\right\rangle_{z, 0}$. The latter are selected by requiring $\left\langle L_{n}^{-1}\right\rangle_{z, 0} \geqslant-21 \mathrm{~m}^{-1}$ for $\tau \in[-200 \mu \mathrm{s}$, $-100 \mu \mathrm{s}]$ for these events. The obtained dynamics is similar to the dynamics of ITEs at probe tip $P_{2}$, where a mode is already clearly visible at $\tau=-160 \mu \mathrm{s}$, Fig. 8(b). This shows that both scenarios of ITEs can be observed at $P_{1}$. With this more restrictive trigger condition, the peak at $P_{1}$, and the moderate value of $\left\langle L_{n}^{-1}\right\rangle_{z, 0}$, which reduces the number of triggers from 3779 to 981 , we have isolated ITEs of the second scenario rather than false triggers. The conditional average $\langle\tilde{n}\rangle_{\text {ca }}$ shown in Sec. III is therefore a mixture of both scenarios. This explains partly its low reproducibility and shows that the knowledge of the conditional average alone can lead to wrong conclusions, as emphasized in a recent work. ${ }^{24}$

We have found a non-negligible number of ITEs at $P_{1}$ that do not involve a strongly negative value of $\left\langle L_{n}^{-1}\right\rangle_{z, 0}$. In the more qualitative analysis of the next section, we show for the similar scenario of ITEs at $P_{2}$ that there is a steepening of the density profile preceding the ITEs in this case as well. This steepening is discussed in the next section.

\section{ITES AT PROBE TIP $\boldsymbol{P}_{2}$}

As discussed in Sec. IV, ITEs at $P_{1}$ are preceded by a steepening of the density profile. In the CS results for $P_{2}$ in Sec. III, the mode seems to pull slightly back radially before the start of the ITE, producing a steepening of the density profile. Profile steepening could therefore be a possible reason for ITEs at $P_{2}$ as well. However, since there is already a strong mode before the ITE, a radial gradient might not be needed for an increase in cross-field transport. It is possible that the positive structures of the mode behave rather like independent structures (blobs) and it is a change in the vertical shape of the positive crests that triggers the ITE. We try to clarify this by the following approach. We select times $\left\{t_{k}\right\}_{k=1}^{N}$ in the discharges at which the mode has a given phase along the $z$ direction, i.e., at which the positive and negative wave structures are at a given vertical position. Among these $N$ events, we select a subseries of $N^{\prime}$ triggers at $\left\{t_{k_{i}}\right\}_{i=1}^{N^{\prime}}$ which are followed by an ITE at $P_{2}$. Then we compare the 2D evolution of $\tilde{n}$ averaged over the $N$ and $N^{\prime}$ events, respectively, to study how their dynamics, starting from similar initial profiles, differ as we proceed in time.

As a starting point, we take the mode at a phase as in frame 11 of Fig. 3, i.e., we take the corresponding conditional average $\langle\tilde{n}\rangle_{\mathrm{ca}}\left(\mathbf{x}, \tau_{0}\right)$ at $\tau_{0}=-204 \mu \mathrm{s}$. The result of the following analysis does not change significantly if we choose a different time $\tau_{0}$ before the radial elongation of the mode in $\langle\widetilde{n}\rangle_{\text {ca. }}$. For each time sample, we evaluate the spatial correlation $\operatorname{corr}\left(t, \tau_{0}\right)$ between the raw signal $\tilde{n}(\mathbf{x}, t)$ and $\langle\widetilde{n}\rangle_{\mathrm{ca}}\left(\mathbf{x}, \tau_{0}\right)$,

$$
\operatorname{corr}\left(t, \tau_{0}\right) \equiv \frac{\sum_{i=1}^{\# \text { tips }} \widetilde{n}\left(\mathbf{x}_{i}, t\right) \times\langle\widetilde{n}\rangle_{\mathrm{ca}}\left(\mathbf{x}_{i}, \tau_{0}\right)}{\sqrt{\left(\sum_{i=1}^{\# \text { tips }} \widetilde{n}\left(\mathbf{x}_{i}, t\right)^{2}\right)\left(\sum_{i=1}^{\# \text { tips }}\langle\widetilde{n}\rangle_{\mathrm{ca}}\left(\mathbf{x}_{i}, \tau_{0}\right)^{2}\right)}},
$$

where the sums are performed over all probe tips of HEXTIP. The quantity $\operatorname{corr}\left(t, \tau_{0}\right)$ is a scalar function bounded between -1 and 1 . It is dominated by fluctuations in the frequency range of the mode and it assumes high values if the phase of the mode at time $t$ coincides with the one in $\langle\widetilde{n}\rangle_{\mathrm{ca}}\left(\mathbf{x}, \tau_{0}\right)$. The times $\left\{t_{k}\right\}_{k=1}^{N}$ are selected by the requirement that $\operatorname{corr}\left(t_{k}, \tau_{0}\right)$ is a local maximum exceeding 0.5 for each $k$.

The subseries $\left\{t_{k_{i}}\right\}_{i=1}^{N^{\prime}}$ of times that correspond to ITEs are selected by the requirement that the signal $\tilde{n}_{P_{2}}$ at probe tip $P_{2}$ assume a maximum value of at least $1.3 \times 10^{16} \mathrm{~m}^{-3}$ for $t \in\left[t_{k}+160 \mu \mathrm{s}, t_{k}+240 \mu \mathrm{s}\right]$. This additional constraint reduces the number of detected events from $\approx 47000$ to $\approx 1700$, showing that the latter are rare events.

Now the conditional averages of the events triggered at $\left\{t_{k}\right\}_{k=1}^{N}$ and $\left\{t_{k_{i}}\right\}_{i=1}^{N^{\prime}}$ are evaluated. We denote them as $\langle\widetilde{n}\rangle_{\mathrm{ca}}^{\text {mode }}(\mathbf{x}, \tau)$ and $\langle\widetilde{n}\rangle_{\mathrm{ca}}^{\mathrm{ITE}}(\mathbf{x}, \tau)$, respectively. Here, $\tau=0$ indicates the time of the CS evolution at which the imposed 

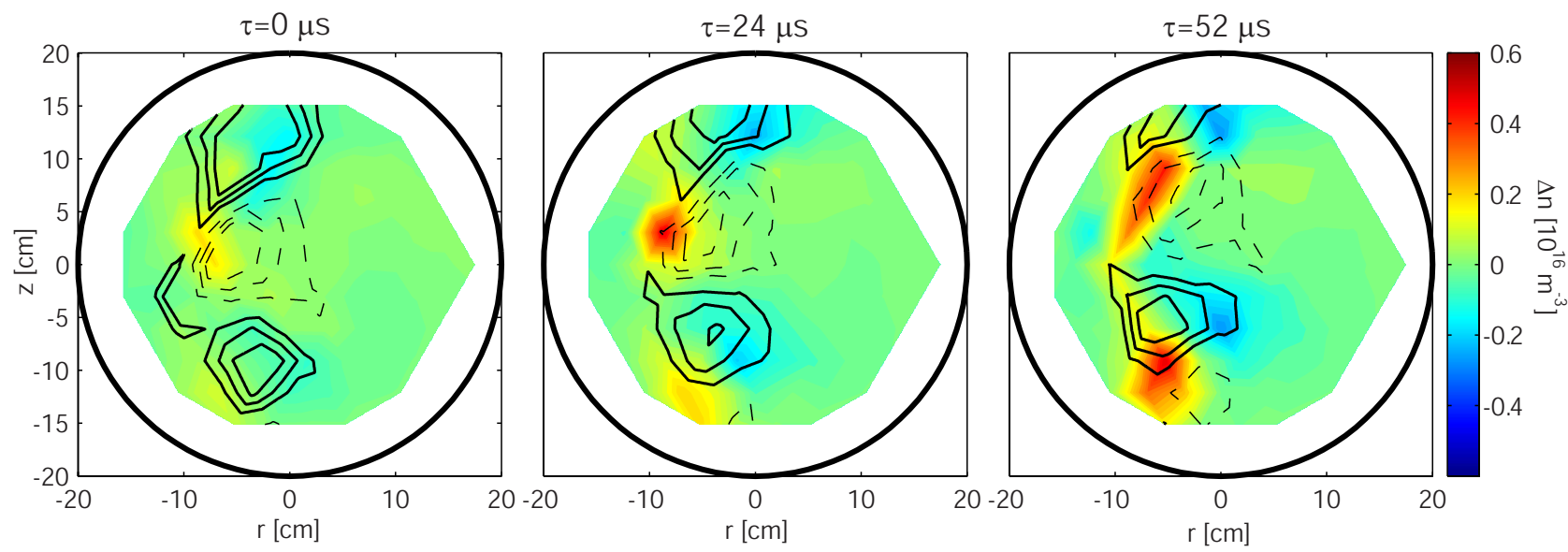

FIG. 9. (Color online) Difference $\langle\widetilde{n}\rangle_{\mathrm{ca}}^{\mathrm{TTE}}-\langle\widetilde{n}\rangle_{\mathrm{ca}}^{\text {mode }}$ between ITEs detected at $P_{2}$ and the average evolution of the mode at three different times $\tau$. Here, $\tau=0$ indicates the time of the CS evolution at which the imposed condition on the phase of the mode is satisfied. In $\langle\tilde{n}\rangle_{\mathrm{ca}}^{\mathrm{ITE}}$, the ITE reaches $P_{2}$ at $\tau \approx 200 \mu \mathrm{s}$. Also shown are the positive (solid lines) and the negative (dashed lines) contours of $\langle\widetilde{n}\rangle_{\mathrm{ca}}^{\mathrm{ITE}}$.

condition on the phase of the mode is satisfied. While $\langle\widetilde{n}\rangle_{\mathrm{ca}}^{\text {mode }}$ shows a mode that is being convected upwards, $\langle\widetilde{n}\rangle_{\mathrm{ca}}^{\mathrm{TTE}}$ is very similar to the CS result from Sec. III. The two conditional averages are compared in Fig. 9, where $\langle\widetilde{n}\rangle_{\mathrm{ca}}^{\mathrm{ITE}}-\langle\tilde{n}\rangle_{\mathrm{ca}}^{\mathrm{mode}}$ as well as the contours of $\langle\tilde{n}\rangle_{\mathrm{ca}}^{\mathrm{TTE}}$ are plotted for three different values of $\tau$. At $\tau=0$, the difference between $\left\langle\left.\widetilde{n}\right|_{\mathrm{ca}} ^{\text {mode }}\right.$ and $\langle\widetilde{n}\rangle_{\mathrm{ca}}^{\mathrm{ITE}}$ is small. Later in time, but still before the start of the ITE, a clear difference builds up. Negative values of $\langle\widetilde{n}\rangle_{\mathrm{ca}}^{\mathrm{ITE}}-\langle\widetilde{n}\rangle_{\mathrm{ca}}^{\text {mode }}$ build up on the LFS of positive wave crests of $\langle\widetilde{n}\rangle_{\mathrm{ca}}^{\mathrm{ITE}}$ and positive values on the HFS of its negative wave crests. Therefore, with respect to its average evolution, the mode shifts to the HFS before an ITE at P2. There is no indication that the vertical shape of the positive wave structure drastically changes. No vertical narrowing can be seen, for example. This strongly suggests that the pulling back of the mode that could already be guessed from the CS result of Sec. III and the profile steepening that goes along with it is at the origin of ITEs at $P_{2}$.

\section{SUMMARY}

In the investigated plasma configuration, intermittent cross-field particle transport events (ITEs) are due to the radial elongation of a large-amplitude interchange wave. Intermittent events are detected at a probe tip on the LFS either as detached structures (blobs) or as radially elongated structures.

Intermittent events at two vertically separated probe tips were further studied. Conditionally sampled data showed ITEs at the lower probe being preceded by a steep density profile. A novel statistical analysis technique was introduced and applied, revealing a monotonic relation between the vertically averaged inverse radial density scale length and the probability for a subsequent ITE.

For a fraction of ITEs at the lower probe, as well as for those at the upper probe, a coherent signal was obtained in the conditional average much earlier than for typical ITEs at the lower probe. The mode does not elongate radially at first, but it rather seems to pull back before it elongates radially at a later time. This pulling back, which implies a steepening of the density profile, was confirmed by comparing ITEs to the average evolution of the mode. These results show that ITEs occur in response to a steepening of the density profile. The difference in the dynamics for ITEs detected at different vertical positions on the LFS indicates possible 3D effects of ITEs. More specific experiments are needed to explore this point.

Being able to predict the occurrence of ITEs could allow us to find ways to avoid them by a real time control system. With the probability comparison chart, we have introduced a generally applicable tool that allows us to qualitatively evaluate the probability for an ITE after the fulfillment of a condition on the plasma profile, such as the strongly negative values of the vertically averaged inverse radial density scale length in the present case.

The presented experimental observations may provide a benchmark for numerical simulations of TORPEX plasmas. We plan to investigate to what extent three-field simulations of interchange turbulence of TORPEX configuration ${ }^{25}$ are able to reproduce the dynamics described in the present paper.

\section{ACKNOWLEDGMENTS}

We thank F. Skiff for useful discussions.

This work is partly supported by the Fonds National Suisse de la Recherche Scientifique. P.R. is supported by a EURATOM Fusion fellowship.

${ }^{1}$ J. A. Boedo, D. L. Rudakov, R. A. Moyer, G. R. McKee, R. L. Colchin, M. J. Schaffer, P. G. Stangeby, W. P. West, S. L. Allen, T. E. Evans, R. J. Fonck, E. M. Hollmann, S. Krasheninnikov, A. W. Leonard, W. Nevins, M. A. Mahdavi, G. D. Porter, G. R. Tynan, D. G. Whyte, and X. Xu, Phys. Plasmas 10, 1670 (2003).

${ }^{2}$ J. L. Terry, S. J. Zweben, K. Hallatschek, B. LaBombard, R. J. Maqueda, B. Bai, C. J. Boswell, M. Greenwald, D. Kopon, W. M. Nevins, C. S. Pitcher, B. N. Rogers, D. P. Stotler, and X. Q. Xu, Phys. Plasmas 10, 1739 (2003).

${ }^{3}$ S. J. Zweben, R. J. Maqueda, J. L. Terry, T. Munsat, J. R. Myra, D. D'Ippolito, D. A. Russell, J. A. Krommes, B. LeBlanc, T. Stolzfus-Dueck, D. P. Stotler, K. M. Williams, C. E. Bush, R. Maingi, O. Grulke, S. A. Sabbagh, and A. E. White, Phys. Plasmas 13, 056114 (2006).

${ }^{4}$ J. R. Myra, D. A. D’Ippolito, D. P. Stotler, S. J. Zweben, B. P. LeBlanc, J. 
E. Menard, R. J. Maqueda, and J. Boedo, Phys. Plasmas 13, 092509 (2006).

${ }^{5}$ O. E. Garcia, J. Horacek, R. A. Pitts, A. H. Nielsen, W. Fundamenski, J. P. Graves, V. Naulin, and J. Juul Rasmussen, Plasma Phys. Controlled Fusion 48, L1 (2006).

${ }^{6}$ M. Spolaore, V. Antoni, E. Spada, H. Bergsaker, R. Cavazzana, J. R. Drake, E. Martines, G. Regnoli, G. Serianni, and N. Vianello, Phys. Rev. Lett. 93, 215003 (2004).

${ }^{7}$ R. Sánchez, B. P. van Milligen, D. E. Newman, and B. A. Carreras, Phys. Rev. Lett. 90, 185005 (2003).

${ }^{8}$ G. Y. Antar, G. Counsell, Y. Yu, B. Labombard, and P. Devynck, Phys. Plasmas 10, 419 (2003).

${ }^{9}$ G. Y. Antar, S. I. Krasheninnikov, P. Devynck, R. P. Doerner, E. M. Hollmann, J. A. Boedo, S. C. Luckhardt, and R. W. Conn, Phys. Rev. Lett. 87, 065001 (2001).

${ }^{10}$ T. A. Carter, Phys. Plasmas 13, 010701 (2006).

${ }^{11}$ T. Windisch, O. Grulke, and T. Klinger, Phys. Plasmas 13, 122303 (2006).

${ }^{12}$ A. Fasoli, B. Labit, M. McGrath, S. H. Müller, G. Plyushchev, M. Podestà, and F. M. Poli, Phys. Plasmas 13, 055902 (2006).

${ }^{13}$ S. H. Müller, A. Diallo, A. Fasoli, I. Furno, B. Labit, M. Podestà, and F. M. Poli, Phys. Plasmas 14, 110704 (2007).

${ }^{14}$ S. H. Müller, A. Fasoli, B. Labit, M. McGrath, O. Pisaturo, G. Plyushchev, M. Podestà, and F. M. Poli, Phys. Plasmas 12, 090906 (2005).

${ }^{15}$ I. Furno, B. Labit, M. Podestà, A. Fasoli, S. H. Müller, F. M. Poli, P. Ricci,
C. Theiler, S. Brunner, A. Diallo, and J. Graves, Phys. Rev. Lett. 100, 055004 (2008); I. Furno, B. Labit, A. Fasoli, F. M. Poli, P. Ricci, C. Theiler, S. Brunner, A. Diallo, J. P. Graves, M. Podestà, and S. H. Müller, Phys. Plasmas 15, 055903 (2008).

${ }^{16}$ H. Johnsen, H. L. Pecseli, and J. Trulsen, Phys. Fluids 30, 2239 (1987).

${ }^{17}$ M. Podestà, A. Fasoli, B. Labit, M. McGrath, S. H. Müller, and F. M. Poli, Plasma Phys. Controlled Fusion 47, 1989 (2005).

${ }^{18}$ F. M. Poli, S. Brunner, A. Diallo, A. Fasoli, I. Furno, B. Labit, S. H. Müller, G. Plyushchev, and M. Podestà, Phys. Plasmas 13, 102104 (2006).

${ }^{19}$ F. M. Poli, P. Ricci, A. Fasoli, and M. Podestà, Phys. Plasmas 15, 032104 (2008).

${ }^{20}$ F. J. Øynes, O.-M. Olsen, H. L. Pécseli, A. Fredriksen, and K. Rypdal, Phys. Rev. E 57, 2242 (1998).

${ }^{21}$ S. I. Krasheninnikov, D. A. D'Ippolito, and J. R. Myra, "Recent theoretical progress in understanding coherent structures in edge and SOL turbulence," J. Plasma Phys. (to be published).

${ }^{22}$ Y. Sarazin and P. Ghendrih, Phys. Plasmas 5, 4214 (1998).

${ }^{23}$ D. Block, I. Teliban, F. Greiner, and A. Piel, Phys. Scr., T T122, 25 (2006).

${ }^{24}$ D. Block, I. Teliban, and A. Piel, Plasma Phys. Controlled Fusion 49, 1707 (2007).

${ }^{25}$ P. Ricci, B. Rogers, and S. Brunner, "High and low confinement modes in simple magnetized toroidal plasmas," Phys. Rev. Lett. (submitted). 